J Hand Surg Eur Vol. 2015 February ; 40(2): 171-176. doi:10.1177/1753193414535720.

\title{
Genetic and environmental influences in Dupuytren's disease: A study of 30,330 Danish twin pairs
}

\author{
S. Larsen ${ }^{1}$, D. G. Krogsgaard ${ }^{1}$, L. Aagaard Larsen², M. lachina ${ }^{2}$, A. Skytthe ${ }^{2}$, and H. \\ Frederiksen ${ }^{3}$ \\ ${ }^{1}$ Department of Orthopaedics, Odense University Hospital, Odense, Denmark \\ ${ }^{2}$ The Danish Twin Registry, Epidemiology Unit, University of Southern Denmark, Denmark \\ ${ }^{3}$ Department of Haematology, Odense University Hospital, Odense, Denmark
}

\begin{abstract}
We aimed to assess the relative contribution of genes and environment in the aetiology of Dupuytren's disease by studying Danish twins born between 1870 and 2000. Twins with a diagnosis $(n=365)$ and the subgroup who also had an operation $(n=259)$ after 1977 were identified through linkage with a nationwide hospital registry among 30,330 monozygotic and same-sexed dizygotic twin pairs. Since monozygotic twins share all their genes and dizygotic twins share on average half of their genetic material, greater phenotypic similarity is expected in monozygotic than in dizygotic twins if a genetic component is involved. The number of concordant male twin pairs with Dupuytren's disease was 17 and 7 (monozygotic and dizygotic pairs, respectively), compared with 60 and 174 discordant monozygotic and dizygotic pairs, yielding probandwise concordance rates of 0.37 (95\% confidence interval (CI): 0.26 to 0.50 ) and 0.07 (95\% CI: 0.04 to 0.14 ), respectively. The heritability of Dupuytren's disease was approximately $80 \%$. We conclude that genetic factors play a major role in the development of Dupuytren's disease.
\end{abstract}

\section{Level of evidence-3}

\section{Keywords}

Dupuytren's disease; genetic; hereditary; twin study

\section{Introduction}

A familial clustering of Dupuytren's disease (DD) has long been recognized and for many years DD has been thought to be inherited as an autosomal dominant condition with incomplete penetrance (Burge, 1999; Hu et al., 2005). However, no single gene has been confirmed to contribute to DD by a fully elucidated mechanism, and it is suggested that DD

Reprints and permissions: sagepub.co.uk/journalsPermissions.nav

Corresponding author: D. G. Krogsgaard, Department of Orthopaedics, Unit for Hand Surgery, Odense University Hospital, Sdr. Boulevard 29, DK-5000 Odense C, Denmark. dkrogsgaard@hotmail.com.

Conflict of interests

None declared. 
has a complex aetiology, arising from environmental and multiple genetic factors (Dolmans et al., 2011); in this type of genetic predisposition, the occurrence of the disease depends on the simultaneous presence of multiple alleles.

The influence of genetic factors in DD has been assessed through familial clustering (Hu et al., 2005), population studies (Finsen et al., 2002) and molecular genetics (Dolmans et al., 2011). In two recent studies the risk of DD was found to be between 2.9 and 4.5 times higher in individuals with an affected sibling than in the general population (Capstick et al., 2013; Hindocha et al., 2006). A positive family history was noted in $41 \%$ and $47 \%$ of patients with DD, respectively. During the last decade, a large number of candidate genes potentially involved in DD have been reported through profiling studies investigating DD at whole-genomic, transcriptomic and proteomic levels (Shih et al., 2012). A whole genome association study conducted among 960 Dutch DD patients and 3117 controls reported nine susceptibility loci, six of them containing genes that are involved in the Wnt signalling pathway (Dolmans et al., 2011). A case control study conducted among 300 DD patients and 300 controls found a statistically significantly higher prevalence of two variants of the dihydrodiol dehydrogenase gene in a group with a positive familial history of DD, in comparison with a group with a negative familial history (Zyluk et al., 2013). Several studies suggest that environmental risk factors, such as alcohol, smoking and diabetes mellitus, are associated with DD (Burke et al., 2007; Geoghegan et al., 2004; Gudmundsson et al., 2000). However, it remains unknown to what extent genetic susceptibility and environmental factors contribute to the development of DD.

Family studies indicating a genetic transmission mode can only provide the upper limit for the heritability (the proportion of the variance attributable to genetic factors), as families share not only genes but also the same environment. Twin studies provide an opportunity to disentangle the effects of genes and environment in a disease. A higher degree of similarity for a trait in monozygotic (MZ) twin pairs compared with dizygotic (DZ) twin pairs indicates a genetic contribution to the aetiology. Previous twin studies of DD comprise only four case studies, with one to two MZ twin pairs in each (Burge, 1999; Lyall, 1993). Using two nationwide registers we have studied 30,330 twin pairs to assess the relative contribution of genes and the environment in the aetiology of DD through heritability analyses.

\section{Methods}

We carried out a register linkage study among Danish twins born between 1870 and 2000. The twins were identified through the population-based Danish Twin Registry (Skytthe et al., 2011). All Danish citizens have a unique and permanent personal identification number (PID) recorded in the Danish Civil Registration System (Pedersen, 2011). By means of the PID, all twins with DD were identified through linkage with the Danish National Patient Register (DNPR) (Lynge et al., 2011).

\section{Study population}

The Danish Twin Registry is nationwide and population based. The registry was established in 1954 and comprises twins born between 1870 and 1930 and surviving for 6 years, as well 
as the birth cohorts 1931-1952, 1953-1982 and 1983-present time that have been included in the registry at later time points. It now includes more than 140 birth cohorts of Danish twins (Skytthe et al., 2002, 2011). Zygosity was established through a questionnaire about the degree of similarity between the twins in a pair. The validity of this zygosity classification has been evaluated by comparison with DNA markers and the misclassification rate has been found to be less than 5\% (Christiansen et al., 2003).

\section{Case ascertainment}

The DNPR was established in 1977 and comprises information on all discharges from all Danish hospitals (Lynge et al., 2011). This register includes the days of admission and discharge, the hospital, the department, as well as up to 20 discharge diagnoses and up to six operations per diagnosis code (Lynge et al., 2011). The diagnoses are classified according to the International Classification of Disease (ICD) using the 8th (1977-1994) and 10th (1994present) revisions. The operations have been classified according to a national classification system during the period 1977-1996; from 1996 the Danish edition of the Nordic MedicoStatistical Committee of Surgical Procedures (NCSP) (Andersen et al., 1999) has been used. The twins with DD were identified through ICD and operation codes. The included diagnosis codes were 733.90 from the ICD 8 and M72.0 from the ICD 10. Operation codes were 7840, 78629, 78529 from the period 1977-1996 and KNDM19 and KNDM09 from 1996 onwards. Data from the DNPR were available for the period 1 January 1977 to 10 October 2010. From the twin pairs in which both were alive on 1 January 1977, we eliminated twins with unknown zygosity and DZ twins of opposite sex, and identified all those with a DD diagnosis $(n=365), 259$ of whom also had a DD operation (Figure 1). Analyses were stratified according to sex, since DD is rare among women, and genetic and environmental factors may play different roles in men and women.

\section{Analyses of twin similarity}

The similarity in MZ and DZ twins was assessed using probandwise concordance rates, odds ratios and correlations for DD. The classic twin methodology is based on the fact that MZ twins share all their genes, whereas DZ twins share on average half of their genes and are no more genetically related than ordinary siblings. A greater phenotypic similarity in MZ twins than in DZ twins is to be expected if there is a significant genetic component in the aetiology of the disease.

The probandwise concordance rate is defined as the proportion of affected twin partners of probands. It reflects the probability of the disease occurring in a twin given that the partner twin is affected. Thus, it is directly comparable with the risk rates reported for other relatives (McGue, 1992). The odds ratio makes use of the additional information available from concordant non-diseased twin pairs and can be interpreted as the increased risk of DD for one twin given the presence as against the absence of DD in the partner twin (Ramakrishnan et al., 1992). The correlations for DD, expressed as tetrachoric correlations owing to a dichotomous outcome, were estimated using the multifactorial threshold model (Falconer, 1965). This assumes that there is an underlying normally distributed liability (susceptibility) to a disease due to genetic and environmental factors. According to standard biometric practice, assuming no epistasis (interaction between genetic loci), no gene-environment 
interaction or correlation and no assortative mating, the total phenotypic variance $(\mathrm{V})$ can be separated into four variance components $\mathrm{V}=\mathrm{A}+\mathrm{D}+\mathrm{C}+\mathrm{E}$, where A refers to the variance contribution from additive genetic effects (the sum of average effects of alleles within and across loci), D refers to the variance contribution from genetic effects due to dominance (interaction of alleles within loci), $\mathrm{C}$ refers to the variance contribution of shared environmental effects and $\mathrm{E}$ refers to the variance contribution from non-shared environmental effects (Neale and Cardon, 1992). Shared environmental effects (i.e. environmental factors that are shared by reared-together twins, such as prenatal and early shared family influences) are a source of their similarity, whereas non-shared environmental effects (i.e. environmental factors that are not shared by reared-together twins) are a source of their dissimilarity.

The genetic and environmental variance components of liability to DD and the likelihoodbased confidence intervals were estimated by structural equation modelling. The method is described in detail elsewhere (Neale and Cardon, 1992). In the full standard biometric model, D and C cannot be simultaneously estimated. We therefore fitted separate ACE and ADE models. Other, simpler models might explain our data equally well. We therefore also fitted AE, DE, CE and E models.

Each model was evaluated in terms of whether it both fitted the data well (i.e. had a nonsignificant Chi-square goodness-of-fit test statistic) and was parsimonious (i.e. none of the parameters in the model could be deleted without a significant increase in Chi square). The Akaike information criterion (Akaike, 1987), which corresponds to the value of Chi-square minus twice the degrees of freedom, provides a summary index of both fit and parsimony to compare non-nested models. Models with the lowest Akaike information criterion are preferred.

Finally, the heritability of the liability to DD (i.e. the proportion of the total phenotypic variance due to genetic variance) was derived from the best-fitting model. The analyses were done using the statistical software program Stata version 13 (StataCorp LP, College Station, TX, USA; http://www.stata.com/manuals13/mv.pdf) and the software program R using the Mets package (Holst and Scheike, 2013).

\section{Results}

Tables 1 and 2 show the numbers of concordant twin pairs with and without DD, as well as discordant twin pairs, probandwise concordance rates, odds ratios and tetrachoric correlation coefficients for male and female twin pairs, respectively. All these statistics are consistently higher in the MZ twin pairs than in the DZ twin pairs, indicating heritable effects. The differences between $\mathrm{MZ}$ and $\mathrm{DZ}$ probandwise concordance rates in male twin pairs were modest compared with the differences in odds ratios and tetrachoric correlation coefficients (Table 1). This is due to the fact that concordance rates do not take into account concordant pairs without DD.

Structural-equation analyses revealed that the best fitting model attributed variation in the liability to DD entirely due to additive genetic and non-shared environmental factors (AE 
model), regardless of sex and whether cases were identified through operation code or diagnosis code. Thus, both the dominance (D) and the shared environment (C) factors were not needed to account for the observed data. According to this model $80 \%$ (95\% confidence interval (CI), 69\% to $87 \%$ ) of the liability to DD in males could be explained by additive genetic effects (the heritability) for DD cases defined by a diagnosis code, or 82\% (95\% CI, $71 \%$ to $90 \%$ ) for DD cases defined by a diagnosis + operation code. Non-shared environmental effects accounted for $20 \%$ (95\% CI, $13 \%$ to $31 \%$ ) or $18 \%$ (95\% CI, $10 \%$ to $29 \%$ ) of the liability to DD for cases identified through diagnosis codes or diagnosis + operation codes, respectively. An overlap of CIs was not seen in the statistics for male twins. A likelihood ratio test showed a significant difference in the tetrachoric correlation coefficient between MZ and DZ twins with a diagnosis $(p<0.001)$ and a diagnosis + operation $(p<0.001)$ (Table 1$)$.

Among females the heritability of DD was similar. The results for female twin pairs were estimated with low precision since only two concordant female MZ pairs and three concordant female DZ pairs were included (Table 2). An overlap of CIs for the probandwise concordance rate, odds ratio and tetrachoric correlation between $\mathrm{MZ}$ and $\mathrm{DZ}$ female twin pairs was seen.

Furthermore, a likelihood ratio test showed that there was no significant difference in the tetrachoric correlation between female MZ and DZ twins with a diagnosis $(p=0.15)$. The difference in the tetrachoric correlation between $\mathrm{MZ}$ and $\mathrm{DZ}$ with a diagnosis + operation was borderline significant $(p=0.058)$ (Table 2).

\section{Discussion}

This study is the first classical twin study on DD and investigates the relative contribution of genes and environment in the development of DD among twins. The results indicate a strong genetic influence in $\mathrm{DD}$, which agrees with observations made in earlier family studies (Capstick et al., 2013; Hindocha et al., 2006; Hu et al., 2005). The advantage of the present study was the use of large nationwide population-based registers with long and complete follow-ups. Cases were identified based on diagnosis codes and operation codes and thus were free of recall bias.

Despite its large size and complete follow-up, our study also has limitations. A limitation was the possibility of diagnostic misclassification, which is a concern in studies that use register diagnoses. Diagnoses and operations from orthopaedic surgical departments in the DNPR have previously been found to be in agreement with information abstracted from medical records in $90 \%$ of the cases (Andersen et al., 1999). Furthermore, it is unlikely that misclassification of DD cases in the registry is dependent on zygosity, and misclassification will therefore contribute to a bias towards finding no difference between the two zygosity groups. Consequently, our estimates of genetic effects are probably conservative.

Information on other risk factors for DD, such as alcohol, smoking and diabetes, was not available. Therefore, we cannot exclude the possibility of confounding as a result of unequal distributions of other DD risk factors between MZ and DZ co-twins. However, if any of 
these risk factors were generally more common in $\mathrm{MZ}$ than in $\mathrm{DZ}$ twins, a higher overall mortality rate in MZ would be expected. In a previous study, mortality from 6 to 90 years of age was found to be similar in MZ twins, DZ twins and the general population, suggesting that diseases and life style factors that are common causes of death are no more common in MZ twins than in DZ twins or singletons (Christensen et al., 1995). Similarly, we found that the occurrence of DD diagnoses was of the same magnitude in both zygosities for each sex (Figure 1). Consequently, substantial differences in the distribution of risk factors for DD between MZ and DZ twins seem unlikely.

Our study shows that genetic effects play a major role in the predisposition to DD in men. The number of women was too small to reach a conclusion on the heritability of DD. Several susceptibility loci or genes have already been identified in DD. Our results show that the search for genetic variants is highly relevant to attain a better understanding of the aetiology and pathogenesis of DD, which could eventually lead to better treatments. However, the aetiology may be complex and involve multiple genetic and environmental factors (Dolmans et al., 2011). This may explain why, to date, no single gene has been confirmed to contribute to DD with a fully elucidated mechanism.

\section{Acknowledgments}

T. Ipsen ${ }^{1}$ and K. Christensen ${ }^{2}$ fulfilled the Vancouver criteria for co-authorship, but are mentioned only in acknowledgements because of the 'six author rule' in the Journal of Hand Surgery.

\section{Funding}

This study was supported by a US National Institute on Aging research grant [NIA-PO1-AG08761]; and the Danish National Research Foundation.

\section{References}

Akaike H. Factor analysis and AIC. Psychometrika. 1987; 52:317-332.

Andersen TF, Madsen M, Jorgensen J, Mellemkjoer L, Olsen JH. The Danish National Hospital Register. A valuable source of data for modern health sciences. Dan Med Bull. 1999; 46:263-268. [PubMed: 10421985]

Burge P. Genetics of Dupuytren's disease. Hand Clin. 1999; 15:63-71. [PubMed: 10050243]

Burke FD, Proud G, Lawson IJ, McGeoch KL, Miles JN. An assessment of the effects of exposure to vibration, smoking, alcohol and diabetes on the prevalence of Dupuytren's disease in 97,537 miners. J Hand Surg Eur. 2007; 32:400-406.

Capstick R, Bragg T, Giele H, Furniss D. Sibling recurrence risk in Dupuytren's disease. J Hand Surg Eur. 2013; 38:424-429.

Christensen K, Vaupel JW, Holm NV, Yashin AI. Mortality among twins after age 6: fetal origins hypothesis versus twin method. BMJ. 1995; 310:432-436. [PubMed: 7873948]

Christiansen L, Frederiksen H, Schousboe K, et al. Age- and sex-differences in the validity of questionnaire-based zygosity in twins. Twin Res. 2003; 6:275-278. [PubMed: 14511432]

Dolmans GH, Werker PM, Hennies HC, et al. Wnt signaling and Dupuytren's disease. N Engl J Med. 2011; 365:307-317. [PubMed: 21732829]

Falconer DS. The inheritance of liability to certain diseases, estimated from the incidence among relatives. Ann Hum Genet. 1965; 29:51-76.

Finsen V, Dalen H, Nesheim J. The prevalence of Dupuytren's disease among 2 different ethnic groups in northern Norway. J Hand Surg Am. 2002; 27:115-117. [PubMed: 11810624] 
Geoghegan JM, Forbes J, Clark DI, Smith C, Hubbard R. Dupuytren's disease risk factors. J Hand Surg Br. 2004; 29:423-426. [PubMed: 15336742]

Gudmundsson KG, Arngrímsson R, Sigfússon N, Björnsson A, Jónsson T. Epidemiology of Dupuytren's disease: clinical, serological, and social assessment. The Reykjavik Study. J Clin Epidemiol. 2000; 53:291-296. [PubMed: 10760640]

Hindocha S, John S, Stanley JK, Watson SJ, Bayat A. The heritability of Dupuytren's disease: familial aggregation and its clinical significance. J Hand Surg Am. 2006; 31:204-210. [PubMed: 16473680]

Holst K, Scheike T. The mets package, ver. 0.2.5, CRAN library, used in the software program R, ver. 3.0.2. $2013 \mathrm{http} / / / \mathrm{www} . c r a n . r-p r o j e c t . o r g$.

Hu FZ, Nystrom A, Ahmed A, et al. Mapping of an autosomal dominant gene for Dupuytren's contracture to chromosome 16q in a Swedish family. Clin Genet. 2005; 68:424-429. [PubMed: 16207209]

Lyall HA. Dupuytren's disease in identical twins. J Hand Surg Br. 1993; 18:368-370. [PubMed: 8345271]

Lynge E, Sandegaard JL, Rebolj M. The Danish National Patient Register. Scand J Public Health. 2011; 39:30-33. [PubMed: 21775347]

McGue M. When assessing twin concordance, use the probandwise not the pairwise rate. Schizophr Bull. 1992; 18:171-176. [PubMed: 1621065]

Neale, MC.; Cardon, LR. Methodology for genetic studies of twins and families. Dordrecht: Kluwer Academic Publishers; 1992.

Pedersen CB. The Danish Civil Registration System. Scand J Public Health. 2011; 39:22-25. [PubMed: 21775345]

Ramakrishnan V, Goldberg J, Henderson WG, et al. Elementary methods for the analysis of dichotomous outcomes in unselected samples of twins. Genet Epidemiol. 1992; 9:273-287. [PubMed: 1398046]

Shih B, Watson S, Bayat A. Whole genome and global expression profiling of Dupuytren's disease: systematic review of findings and future perspectives. Ann Rheum Dis. 2012; 71:1440-1447. [PubMed: 22772327]

Skytthe A, Kyvik KO, Holm N, Vaupel J, Christensen K. The Danish Twin Registry: 127 birth cohorts of twins. Twin Res. 2002; 5:352-357. [PubMed: 12537858]

Skytthe A, Kyvik KO, Holm NV, Christensen K. The Danish Twin Registry. Scand J Public Health. 2011; 39:75-78. [PubMed: 21775358]

Zyluk A, Debniak T, Puchalski P. Common variants of the ALDH2 and DHDH genes and the risk of Dupuytren's disease. J Hand Surg Eur. 2013; 38:430-434. 


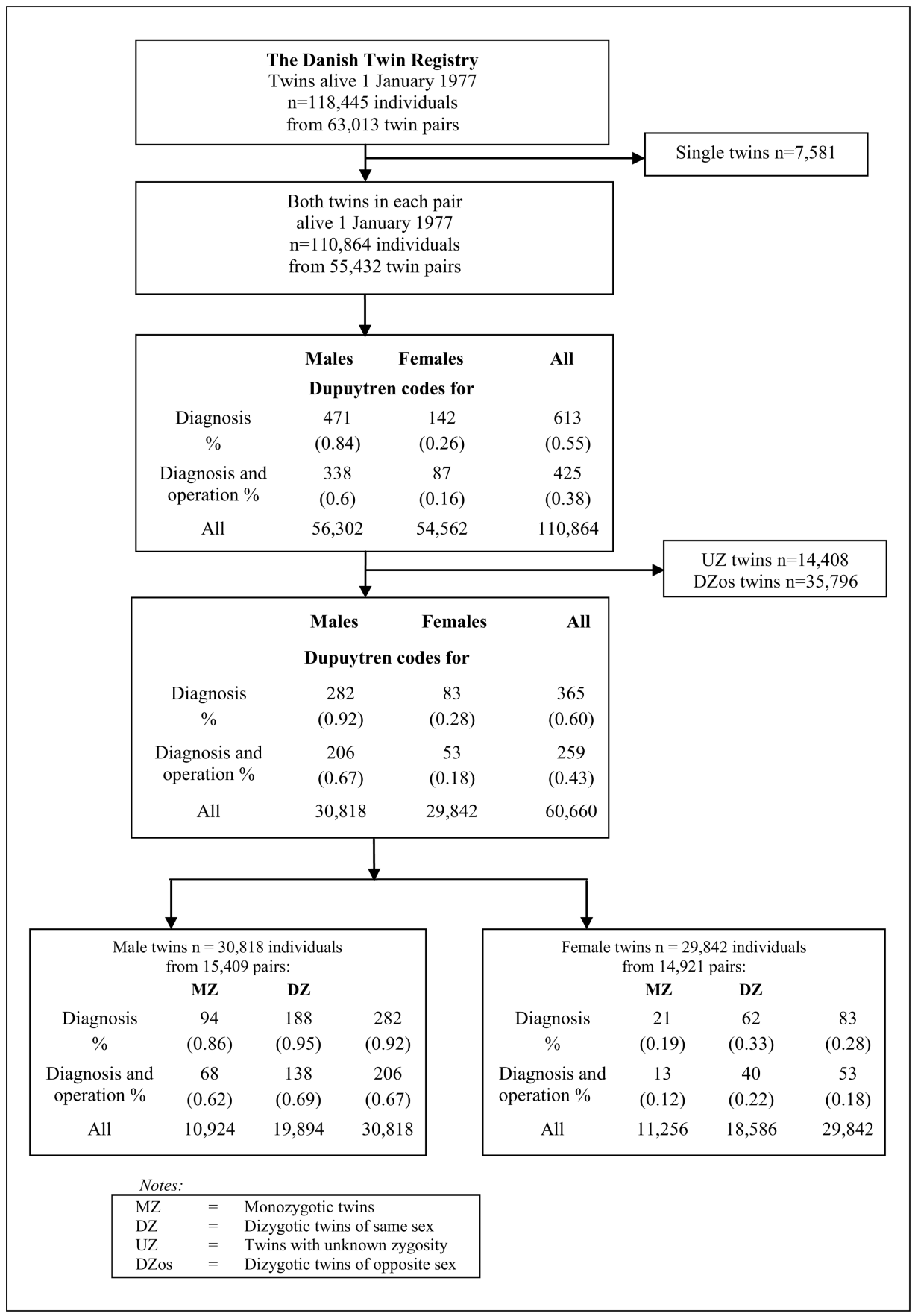

Figure 1.

Flow chart of the study population in the Danish Twin Registry and the DNPR. 

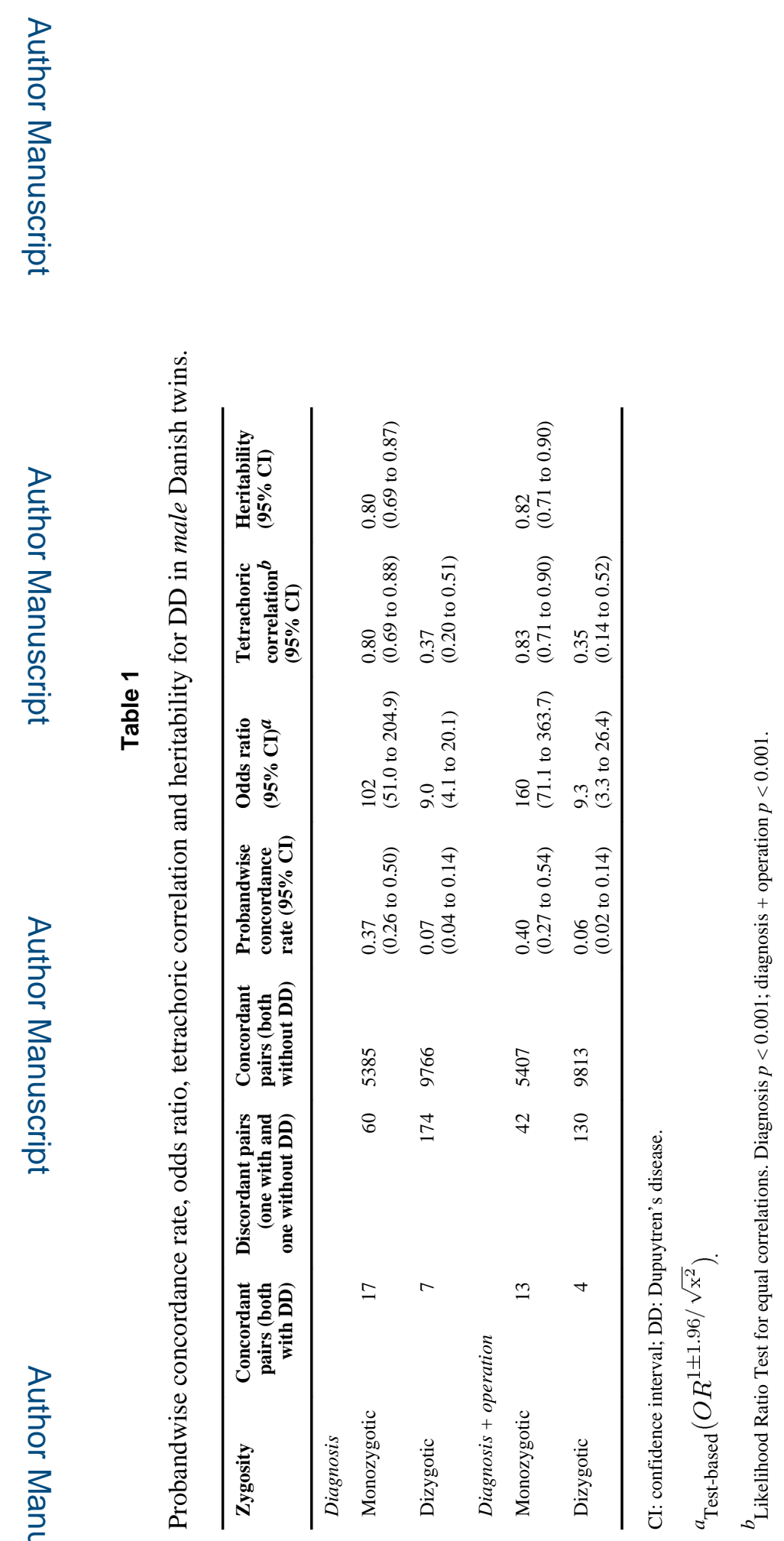

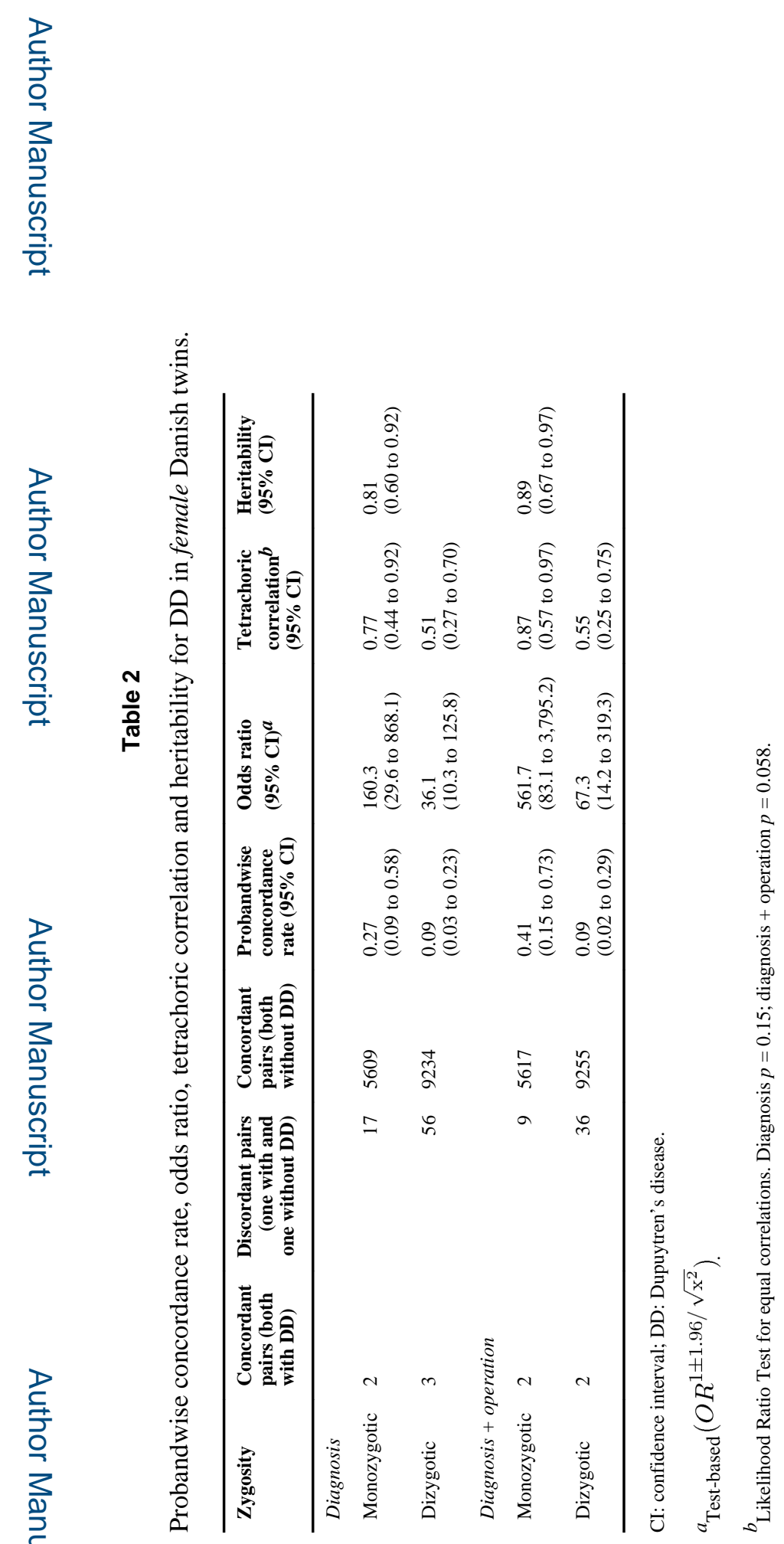